\title{
Magnetic Resonance Imaging, Microscopy, and Spectroscopy of the Central Nervous System in Experimental Animals
}

\author{
Istvan Pirko,* Stanley Thomas Fricke, ${ }^{\dagger}$ Aaron J. Johnson,* \\ Moses Rodriguez, ${ }^{\star}$ and Slobodan I. Macura ${ }^{\S}$ \\ *University of Cincinnati, Department of Neurology, Cincinnati, Ohio 45267; ${ }^{\dagger}$ Georgetown University, Department of \\ Neuroscience, Washington, D.C. 20057; Mayo Clinic College of Medicine, ${ }^{\ddagger}$ Departments of Neurology and Immunology, \\ and ${ }^{\S}$ Department of Biochemistry and Molecular Biology, Rochester, Minnesota 55905
}

\begin{abstract}
Summary: Over the last two decades, microscopic resolution in vivo magnetic resonance imaging (MRI) techniques have been developed and extensively used in the study of animal models of human diseases. Standard MRI methods are frequently used in clinical studies and in the general clinical practice of human neurological diseases. This generates a need for similar studies in experimental animal research. Because small rodents are the most commonly used species as animal models of neurological diseases, the MRI techniques need to be able to provide microscopic resolution and high signal-to-noise ratio images in relatively short time. Small animal MRI systems use very high field-strength magnets, which results in higher signal to noise ratio; however, the contrast characteristics of live tissue are different at these field strengths. In addition to standard MRI techniques, several new
\end{abstract}

applications have been implemented in experimental animals, including diffusion and perfusion studies, MR angiography, functional MRI studies, MRI tractography, proton and phosphorous spectroscopy, cellular and molecular imaging using novel contrast methods. Here we give an overview of how to establish a small animal imaging facility with the goal of CNS imaging. We describe the basic physical processes leading to MR signal generation, highlighting the differences between standard clinical MRI and small animal MRI. Finally, typical findings in the most common neurological disease categories and novel MRI/magnetic resonance spectroscopy methods used in their study are also described. Key Words: Microscopic resolution MRI, MR microscopy, small animal MRI, MR spectroscopy, proton, and phosphorous MRS.

\section{INTRODUCTION}

Animal models are frequently used in the study of complex human diseases. These disease models represent at least a few selected aspects or occasionally the full spectrum of the corresponding human disease. Although there has been success in basic science using cell and tissue cultures, the complexity of a mammal organism cannot be effectively modeled without the use of live animals. Through the use of new technology and the availability of transgenic animals, the molecular mechanism of several diseases can be explored.

One of the most common ways to approach research questions in biomedical sciences is to visualize tissue compartments or cellular interactions in various tissues. A classic method to visualize tissues and cells is by light

Address correspondence and reprint requests to Istvan Pirko, M.D., Assistant Professor of Neurology, University of Cincinnati, Department of Neurology, 231 Albert Sabin Way, ML 0525, Cincinnati, OH 45267-0525. E-mail: Istvan.Pirko@uc.edu. microscopy. This requires ex vivo tissue samples obtained by biopsy or more commonly by sacrificing animals. The maximum resolution in light microscopy is determined by the wavelength of the light source used. With this method, static tissue samples can be studied. However, most disease processes are dynamic. To study a dynamic process with static tissue-based techniques, a large number of animals is needed for every experiment so that some of them can be sacrificed and their tissue processed at given time points of interest. Looking at the kinetics of disease processes using microscopy can often be overwhelming.

Over the last few decades, several new diagnostic imaging techniques have become available to study disease processes in vivo. Many of these imaging modalities expose study subjects to ionizing radiation, so that the absorption or emission patterns can be used to create images. Standard x-rays, CT, PET, and SPECT scans fall into this category. From the image analysis standpoint, some of these techniques can be thought of as "trans- 
illumination" or summed voxel projection techniques. CT, PET, and SPECT can also provide slice-based information, allowing the rendering of three-dimensional (3D) images.

The focus of this review is to describe the methods, the limitations, and the most common applications of small animal magnetic resonance imaging (MRI) in neurological diseases. MRI is a relatively new imaging technique that has evolved from nuclear magnetic resonance (NMR) spectroscopy. The first manuscript describing MRI of a rat was published almost 25 years ago. ${ }^{1} \mathrm{NMR}$ and MRI are some of the most exciting fields of applied physics, being extensively studied by physicists, chemists, biochemists, radiologists, and other life scientists. The number of Nobel prizes awarded to NMR and MRI scientists also emphasizes the importance and recognition this field has received (http://nobelprize.org/). These include the 2003 Nobel Prize for Physiology and Medicine awarded to Paul Lauterbur and Peter Mansfield for the development of MRI. Earlier, NMR related Nobel Prizes were awarded for Chemistry (Kurt Wüthrich in 2002, Richard R. Ernst in 1991) and Physics (Felix Bloch and Edward M. Purcell in 1952 and Isidor I. Rabi in 1944). For a review of the foundations of advanced MRI, PET, and SPECT in humans, the reader is referred to excellent reviews presented separately in the current volume. $^{2-5}$

MRI has the capability of studying live organisms without exposing them to potentially harmful ionizing radiation. Depending on the technique used, slice-based as well as true 3D image sets can be obtained. As discussed later in this review, there are several ways to acquire MRI images, and the different pulse sequences used are key determinants of tissue contrast in MR imaging. Besides anatomical imaging, MRI is also capable of providing physiological information about several important aspects of biological processes. These include circulation and CSF flow, cerebral blood volume distribution, activity mapping with functional (f) MRI or $\mathrm{Mn}^{++}$-based techniques, metabolite distribution with chemical shift imaging, or in vivo $\mathrm{pH}$ measurement via phosphorous magnetic resonance spectroscopy (MRS). MRI is rapidly becoming the tool of choice in the study of several neurological disease models. Because MRI scanning is used as a routine test in the study of neurological diseases, correlation of MRI findings in humans, disease models, and tissue samples can now be done in laboratories equipped with small animal imaging facilities.

\section{The technical aspects of establishing a small animal imaging facility}

Many universities and research institutions now own small animal magnets. The establishment of such a facility is a major undertaking. The cost of a small animal MRI system is in the range of $\$ 800,000-\$ 2,000,000$.
The most commonly used high-field strength magnets are cryomagnets, where the low temperature required for superconductivity is achieved by the use of liquid cooling media. The superconducting core is cooled by liquid helium, whereas the outer parts are cooled by liquid nitrogen. Standard maintenance includes the replacement of the boiled off nitrogen every week and liquid helium every several weeks. Recently, a new technology emerged with closed cycle liquid helium cooling which eliminates or at least greatly reduces the need for cryo liquid refills.

Generally, most facilities employ at least one physicist, one engineer, and several technicians ranging with skills in computer operation and programming, MRI operation, data processing, image analysis, and animal handling. Besides the unique hardware requirement of the scanner, a very sophisticated computer environment is also needed to effectively run the facility. Proprietary software packages have been developed by all system manufacturers (Bruker, Varian, Tecmag, etc.) to run the scanner and to provide basic image and NMR spectrum analysis capabilities. Many researchers also develop macros, pulse program sequences, post-processing, and image analysis programs. Research systems tend to be different from clinical in terms of being more extensively programmable; however, this comes at the expense of possessing only a very few ready-made "foolproof" sequences that can be run without significant fine tuning of all variables in a given experiment.

A frequently forgotten aspect of MR imaging is that it generates a vast amount of data in very little time. It is not unusual for a 3D data set to be hundreds of megabytes to a few gigabytes in size. After an extensive image acquisition session involving multiple animals, investigators frequently find themselves overwhelmed by the amount of data generated. The data processing and analysis required to answer specific research questions usually involves an interaction between the NMR/MRI facility personnel and the investigator. It is unreasonable to expect the MR facility personnel to provide the investigator with specific answers. Generally, the investigators should have a basic understanding of how to read MR images, know the anatomy of the studied species, and have some basic knowledge about the expected imaging findings in the particular disease model of interest. It is worth noting that image processing frequently requires much more time than acquiring the raw data. Some image processing and visualization tools generally are included with the scanner manufacturers' software, but they are generally insufficient to provide the desired answers. Several commercially available biomedical image post-processing software packages are available and many research institutions develop their own software applications for this purpose. Notable examples of image analysis software are Analyze $^{6,7}$, Statistical Parametric Mapping ${ }^{8}$, and NIH image (http://rsb.info.nih.gov/nih-image/Default.html). 


\section{Handling animals in the scanner facility}

MRI requires the study subjects to be immobilized. To achieve this goal, general anesthesia must be used in small animal experiments. ${ }^{9}$ This may include the use of injectable barbiturates, but most researchers prefer to use inhalational anesthesia. Unlike injectable agents, inhalational anesthesia is administered continuously and can be calibrated to the specific requirements posed by the animal and the experiment. Most commonly inhalational isoflurane is used. The investigators have to be aware that different mice strains may need different levels of anesthetics; also, certain strains are prone to develop myoclonic jerks while under isoflurane anesthesia. This may require the use of preventive medications in certain strains of mice. The investigator has to be aware that the anesthesia may alter the desired imaging outcome; this is especially true for cerebral blood volume and functional MRI studies. ${ }^{10}$

While under general anesthesia, the animals are more prone to develop hypothermia. The core temperature inside narrow-bore scanners is in the low $50^{\circ} \mathrm{F}$ range $(15-$ $20^{\circ} \mathrm{C}$ ). Therefore, a heating system should be incorporated in the imaging probe to help maintain the normal core temperature of the animals while in the scanner. This can be done by using warm airflow or by circulating hot water in the probe bed. If circulating water is used, one has to make sure that the heating elements are not included in the field of view because they may generate an undesirably strong signal during proton imaging.

Besides the anesthesia and maintaining a suitable core temperature, another important factor is that probe should keep the animal stable during the imaging session. ${ }^{9-11}$ Most small animal systems are shipped with probes that can accommodate mice or rats. Many labs develop custom-made holders for specific applications (FIG. 1). A good probe will achieve maximal stabilization without any trauma to the animal. Ideally, this should be done in a reproducible and stereotactic way. This will allow for easier data after processing because coregistration will likely not be necessary.

Small rodents can easily be anesthetized for $2-3 \mathrm{~h}$. This is usually enough time to finish most experiments. We have had good success with anesthetizing mice for much longer, up to $10 \mathrm{~h}$. If one has to run very long experiments, adequate hydration should be provided to the animals. This is most often achieved by intraperitoneal injection of normal saline.

While in the scanner, several physiological parameters can and should be monitored. MRI compatible electrodes make electrocardiogram (ECG) monitoring possible. There are several methods for respiratory monitoring, including deriving the respiratory signal from ECG baseline fluctuations, or through a pressure sensor detecting movements of the thorax. Rectal probes can be used to monitor the animal's core temperature.
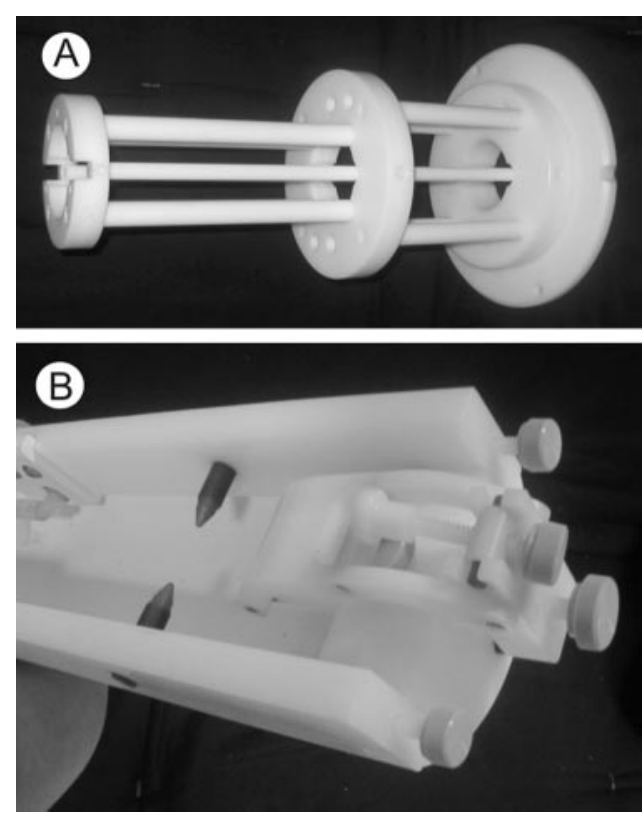

FIG. 1. Custom-designed mouse probe. Mounting plane (A) custom designed for 20-cm bore MRI. Stereotactic device (B) showing ear and teeth bars plus gas anesthetic supply and scavenging lines.

It is equally important to provide appropriate care to animals recovering from the imaging sessions. During the recovery period, we supply the animals with pure oxygen and maintain their core temperature by placing them in a cage equipped with a heater for the first 30-50 $\mathrm{min}$ of the recovery. Animal loss due to imaging and anesthesia is rare but depending on the severity of the disease process studied, it may occasionally occur even with the most careful planning.

\section{Basics of MRI physics}

The full and thorough description of MR physics is far beyond the scope of this review. The separate reviews in the current volume on human $\mathrm{MRI}^{2}$ and $\mathrm{MRS}^{3}$ include some discussion of MRI physics. In addition, several standard textbooks are available discussing this topic. ${ }^{12-14}$ It is important to emphasize that without a basic knowledge of MRI physics, several basic imaging phenomena, including the sources of artifacts would be very difficult for the investigator to understand.

In general, MRI experiments use signals generated by protons, most of which are present in water molecules. Theoretically, many nuclei exert properties of magnetic moment, including ${ }^{1} \mathrm{H},{ }^{31} \mathrm{P},{ }^{13} \mathrm{C},{ }^{15} \mathrm{~N}$, and ${ }^{19} \mathrm{~F}$. Protons are the most abundant nuclei in living organisms and for this reason protons are the most frequently studied nuclei. As a crude simplification, these nuclei can be thought of as small magnets that are spinning. If we look at a biological sample at rest, the orientation of the spins will be random. When we place this sample in a strong external magnetic field (called $\mathrm{B}_{0}$ field in standard NMR 
texts) the spins will align with the field creating a small excess of nuclear magnetization. The sample will thus have a new property, the net magnetization vector (NMV, the added-on magnetization of the spins that align with the $\mathrm{B}_{0}$ field). This net magnetization vector points in the direction of $\mathrm{B}_{0}$, and precesses with the Larmor frequency $(\omega)$. This Larmor frequency is very important, as this denotes the frequency of radio waves that these spinning nuclei can absorb. This frequency is linearly proportional to the gyromagnetic ratio $(\gamma)$ of the nucleus (42.58 MHz/T for protons) and to the external magnetic field $\left(\mathrm{B}_{0}\right)$. The relationship that describes the association of field strength with the precession frequency is the Larmor equation:

$$
\omega=\gamma \mathrm{B}_{0}
$$

If a radiofrequency (RF) pulse of the Larmor frequency is applied to the precessing NMV, with its own magnetic component at a $90^{\circ}$ angle to the NMV, the NMV will absorb energy, and will "flip" down to the transverse plane. This process is commonly referred to as excitation. As soon as the RF pulse is switched off, the NMV will start recovering back to its baseline at the given $\mathrm{B}_{0}$ field strength. This process is called relaxation. The recovery process along the longitudinal axis is called $\mathrm{T} 1$ relaxation, or spin-lattice relaxation. During this process, the nuclei are giving up energy to their immediate surroundings ("lattice"). The relaxation process in the transverse plane (the plane orthogonal to $\mathrm{B}_{0}$ ) is referred to as $\mathrm{T} 2$ relaxation, or spin-spin relaxation. In this process, nuclei exchange energy with each other. If one places an $\mathrm{RF}$ coil in the transverse plane, the transversal component of NMV will induce a current in it as it precesses around $\mathrm{B}_{0}$. This is how the NMR signal is generated. A few commonly used parameters in imaging refer to these processes. TR describes the repetition time and determines how often the excitation cycles are repeated. TE is known as echo time, which describes the time from the application of the RF pulse to the time of signal acquisition. Thus, TR is always longer than TE.

In the above model where nuclei are placed in a homogeneous magnetic field, and an RF excitation pulse is followed by relaxation of the nuclei, the entire sample would only give one signal. In other words, in the above highly simplified system, the NMR signal cannot be spatially resolved. To "split this signal up" to smaller pieces representing small volume units or voxels, the NMR signal needs to be space encoded. This is achieved by an additional magnetic field inside the scanner. This field is parallel to $\mathrm{B}_{0}$ and is called a gradient field because its strength varies with the position. The gradient (change of field by unit change of spatial coordinate) has three components, $G_{x}, G_{y}$, and $G_{z}$ each associated with its respective spatial axis, $x, y$, or $z$. The spins experience different field strengths depending on where they are within this gradient field. Unlike the strong field of the cryomagnet, the gradient fields are much weaker and are generated by electromagnets. These fields are switchable (each component can be turned on and off independently) and configurable by the operator (the noise experienced by MRI study subjects is actually related to gradient switching). Spins in different volume units inside the gradient experience different magnetic field. Each "sub-field" is associated with a different Larmor frequency. This technique of spatial localization carries the name "frequency encoding." If one only uses frequency encoding, MR imaging already is attainable through a technique called back projection. With this method, the MR image is reconstructed from a series of scans with rotated frequency encoding gradients; a very similar method is used for image generation in CT scanners. However, in MRI scanners it is no longer used in the modern era of Fourier-transform MRI. In Fouriertransform MRI, instead of simultaneous switching of two orthogonal gradients (this combination would generate rotation of a single frequency encoded gradient) gradients are switched alternatively. The second gradient is called the phase encoding gradient. ${ }^{15,16}$ The phase encoding gradient applies a specific phase angle to a transverse magnetization vector. While the phase encoding gradient is on, each transverse magnetization vector has its own unique Larmor frequency. When the phase encoding gradient is turned off, the external magnetic field experienced by each spin vector is identical (and equal to $\mathrm{B}_{0}$ ). Therefore, the Larmor frequency of each transverse magnetization also is identical; however, the phase angle of each vector is different. This is because they were spinning with different Larmor frequencies while the phase encoding gradient was on, so when the gradient is switched off, their phases will be "frozen" at different angles. The phase angle is related to the frequency of the spinning while the phase encoding gradient was on and on the duration of the gradient.

By combining slice selection, phase encoding and frequency encoding, one can create imaging pulse sequences. In two-dimensional (2D) experiments, all the above three components are used in an orthogonal manner. In true 3D experiments, two phase and one frequency-encoding direction are used.

\section{Differences between small animal imaging and human MRI}

It is important to understand that the study of experimental animals with MRI differs in more ways than just the smaller scale that rodent imaging requires; however, the smaller scale itself is a major problem to overcome (FIG. 2.)

Most animal magnets operate at very high field strength, in the range of 3-14 Tesla. One Tesla repre- 


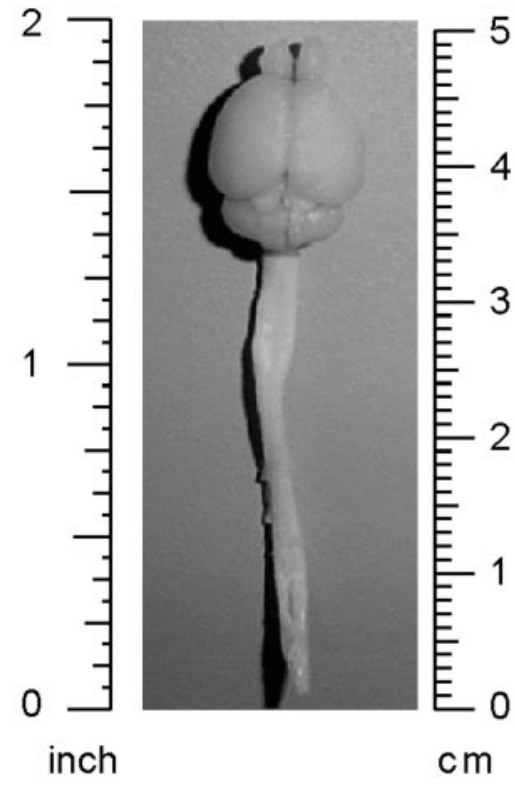

FIG. 2. Extracted brain and spinal cord of a SJL/J mouse. The entire mouse brain is about the size of the caudate nucleus in a human brain.

sents a field strength that is approximately 10,000 times stronger than the magnetic field on the surface of our planet. Most clinical scanners available for routine imaging studies operate in the $0.4-1.5$ Tesla range. To effectively use the very high-field strength animal imaging magnets, the MR investigator has to understand that $\mathrm{T} 1$ and $\mathrm{T} 2$ mechanisms behave very differently at these field strengths. $\mathrm{T} 1$ and $\mathrm{T} 2$ relaxation times are field strength dependent. For example, when investigating gray and white matter differences in the brain, the relaxation times are found to be much closer to each other than what is usually observed in lower field strength human imaging, and therefore the gray-white contrast differentiation is actually worse. One also has to understand that since the $\mathrm{T} 1$ and $\mathrm{T} 2$ processes both require less time, the high TE (echo time) values used in human imaging should not be expected to provide similar results at this field strength.

\section{Signal-to-noise and contrast-to-noise ratios}

One main goal in small animal imaging is to achieve very high spatial resolution. The actual spatial resolution depends on several factors, including the field strength, the abundance of water (or other examined nuclei) in the sample, the imaging technique used ( $\mathrm{T} 1$ weighted, $\mathrm{T} 2$ weighted, etc), the number of signal averages (NEX) and the size of the RF coil. ${ }^{17,18}$

One key parameter describing the quality of MRI images is the SNR, or signal to noise ratio. This ratio is determined by comparing the nontissue voxel elements ("noise") to elements of tissue signal. The SNR depends on the field strength, and it increases almost as the square of the field strength. The SNR also depends on the coil size and the coil filling factor. In general, the smaller the coil and the better it encompasses a given sample, the better the coil filling becomes, and thus the SNR improves. SNR also increases when the signal is averaged by repeated acquisitions. The SNR increases as the square root of the number of averages, for example, four averages would increase the SNR by twofold. While this clearly is the easiest way to increase SNR in a given experiment, the animals may not be able to tolerate very long acquisition sessions. A 30-min-long 3D acquisition of the brain would become $2 \mathrm{~h}$ long, if we wanted to increase the SNR by twofold using this method.
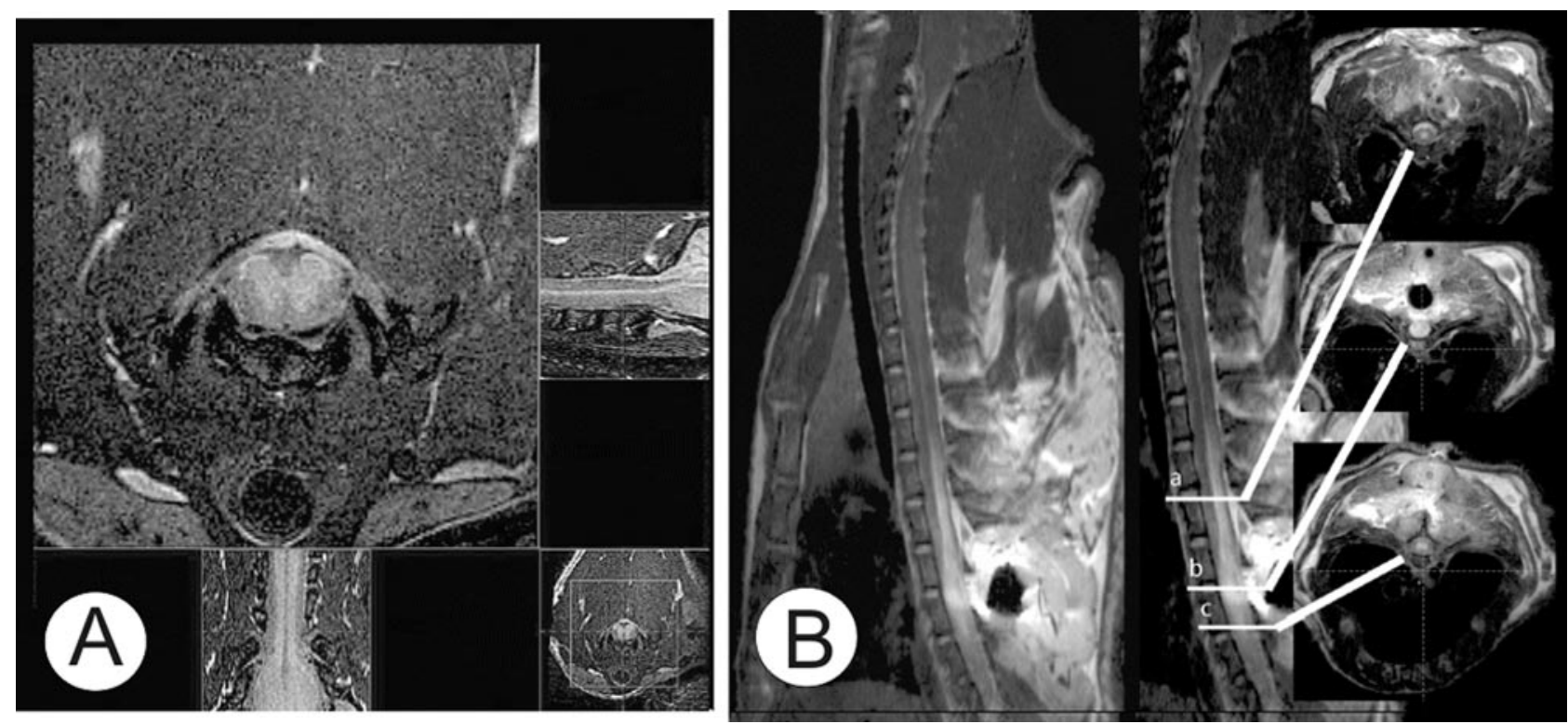

FIG. 3. A: Anatomical imaging of rat spinal cord in vivo. Note the excellent delineation between gray and white matter. B: In vivo rat cord, T2-weighted study. Spinal cord injury is clearly shown on sagittal image, also on axial cuts (a-c) at different levels across the lesion. 

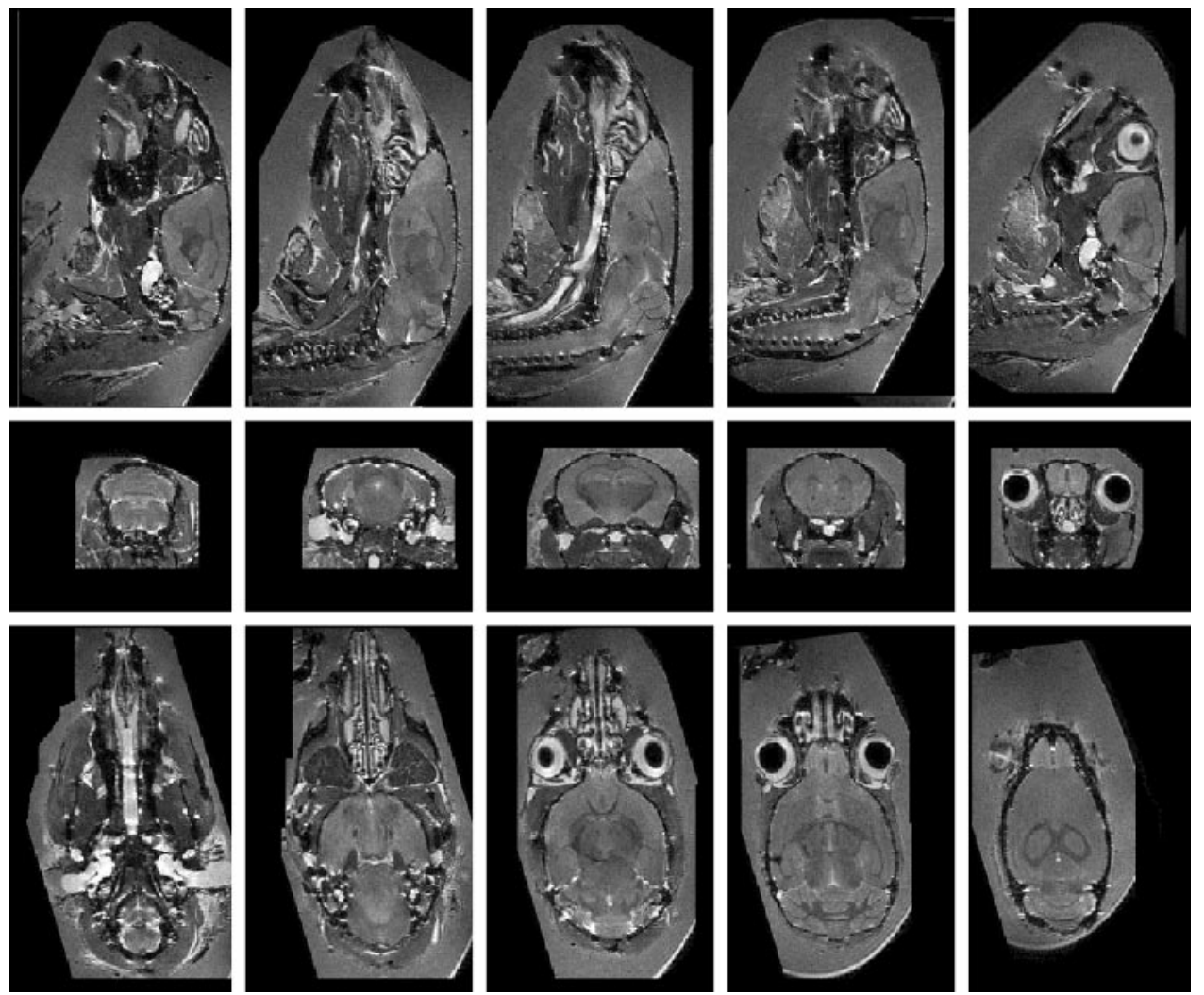

FIG. 4. 7 Tesla ( $300 \mathrm{MHz}$ ) MR microscopy of ex vivo mouse head. Note excellent anatomical resolution of sagittal (top) coronal (middle) and axial (bottom row) images extracted from a 3D data set. The high SNR and high isotropic resolution (100 $\mu \mathrm{m} / \mathrm{pixel})$ are achieved at the expense of long scanning time $(33 \mathrm{~h})$. Other parameters: FOV $51.2 \times 25.6 \times 25.6 \mathrm{~mm}^{3}$, matrix size $512 \times 256 \times 256$, TR $1.8 \mathrm{~s}$, TE $50 \mathrm{~ms}$.

Another important factor to consider is that improving contrast of an image by increasing the TE (allowing for more T2 weighting) will almost always result in lower SNR. One way to find a healthy compromise is to lower the resolution of the image. Lower resolution for the same sample volume means larger voxel sizes, thus the SNR is increased. Halving the in plane resolution of a $2 D$ image will increase the SNR by a factor of 4 and halving the isotropic resolution of a 3D image will increase the SNR by a factor of 8 .

While optimizing the SNR is a very important aspect of MR imaging, a high SNR image in itself does not mean an optimal image. High SNR images may be lacking several features if the contrast-to-noise ratio (CNR) is not optimized. CNR determines how well different structures can be differentiated on a given image. CNR is defined as the difference between the SNR of distinct tissues in the sample (for example, gray and white matter of the brain). It is also important to realize that improving the CNR frequently means that the SNR will in turn decrease. Several contrast mechanisms are applicable in MR imaging. These include differences in relaxation times (T1, T2, and $\mathrm{T} 1 \rho$ ), proton density, diffusion coefficient, chemical exchange rate, etc. The existence of potential mechanisms depends on the sample properties and the one of interest can be emphasized by an appropriately designed pulse sequence. Another way to enhance images is to introduce a contrast agent that will make parts of an image become hyperintense or hypointense depending on the agent used. Elements that are paramagnetic such as $\mathrm{Fe}^{3+}$ (Iron), $\mathrm{Mn}^{2+}$ (Manganese), $\mathrm{Gd}^{2+}$ (Gadolinium), $\mathrm{Ln}^{2+}$ (Lanthanum) are all contrast agents that have a pronounced effect on the outcome of a $\mathrm{T} 1$ or $\mathrm{T} 2 *$-weighted image.

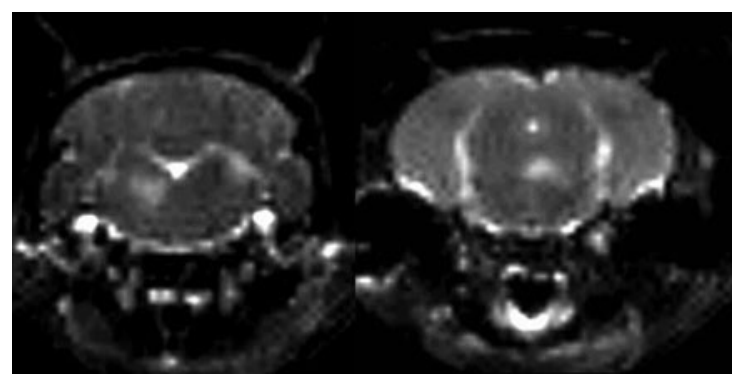

FIG. 5. Demyelinating lesions in interferon- $\gamma$ receptor knockout mice following Theiler's virus infection. Note the high signal intensity areas in the brainstem and near the thalamus on these T2-weighted in vivo images. 


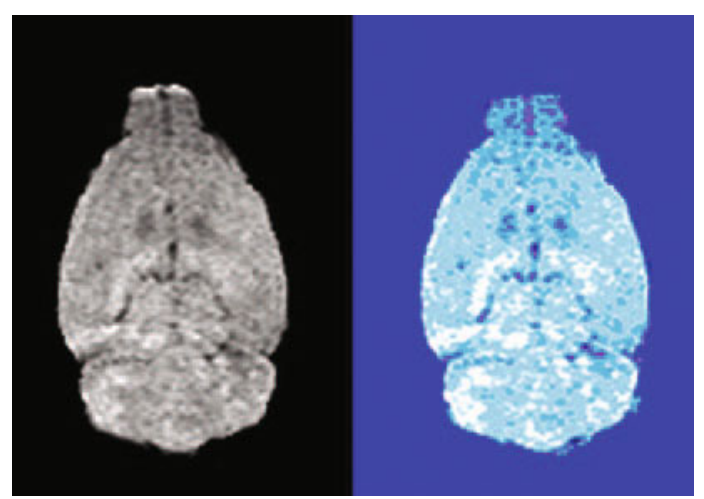

FIG. 6. Cell-specific in vivo MRI of CD-4+ T-cells in SJL/J mouse brain following induction of experimental allergic encephalomyelitis (EAE). Note high signal areas on these T1-weighted images. Right panel shows the same axial cut as left, with tomographic blue encoding to help visualize enhancing areas. The cell-specific labeling was obtained by the use of CD4-specific USPIO-conjugated antibodies.

\section{What determines image quality overall?}

Most investigators would like to obtain image sets of excellent resolution and high SNR in a very little time. It is important to recognize that each of the above three component (SNR, resolution, imaging time) are interdependent. For example, the price one pays for a good SNR is frequently a lower resolution and/or longer acquisition time. Or, if both good SNR and resolution are the goals, then the imaging time must be prolonged. Finding a healthy balance may be a difficult task; several parameters of the studied model should be considered when trying to optimize image acquisition.

\section{Special problems related to high field strength in vivo imaging}

Living organisms can be considered inhomogeneous samples, with compartmentalized organs and parts of organs. Gaseous, solid, and various consistencies of liquid (from fluid to gelatinous) substances can be found in living organisms. These different consistencies have different magnetic susceptibilities, and while this in general does not represent a major problem at lower field strength, it becomes a very important problem at the high field strength used in small animal imaging and MRI microscopy.

Magnetic susceptibility is a known source of artifacts. One of the best known is the loss of brain signal near air-filled sinuses. Gradient echo (GE) imaging is especially prone to these artifacts. It can also interfere with fat suppression and diffusion experiments. Several methods have been proposed to reduce these artifacts, like the gradient echo slice excitation profile imaging (GESEPI) sequence. ${ }^{19,20}$ In GE imaging, slice selection correction methods have also been tried and successfully used. ${ }^{21,22}$ For spin echo multislice studies, the use of phase-phase encoding was found helpful in reducing susceptibility related artifacts. Variants of fast spin echo (FSE) or RARE (rapid acquisition relaxation enhancement) ${ }^{23}$ sequences use RF refocused echoes, which eliminate or reduce much of the susceptibility-related artifacts. It should also be noted that in some applications, susceptibility-related artifacts might be of benefit. The new generation iron oxide based negative contrast agents [small or ultra small superparamagnetic iron oxide particles or (U)SPIO-s] are detectable through their property of generating strong susceptibility effects. Because susceptibility artifacts are stronger at high field strength, these contrast materials are especially suitable for small animal imaging.

\section{MRI of the CNS in small animal models}

Several neurological disease models have been studied with small animal MRI. One of the most basic applications is to generate $3 \mathrm{D}$ anatomical atlases of rodent brains. This has been accomplished by several groups. $^{24-27}$ Similarly, MRI atlases describing mouse

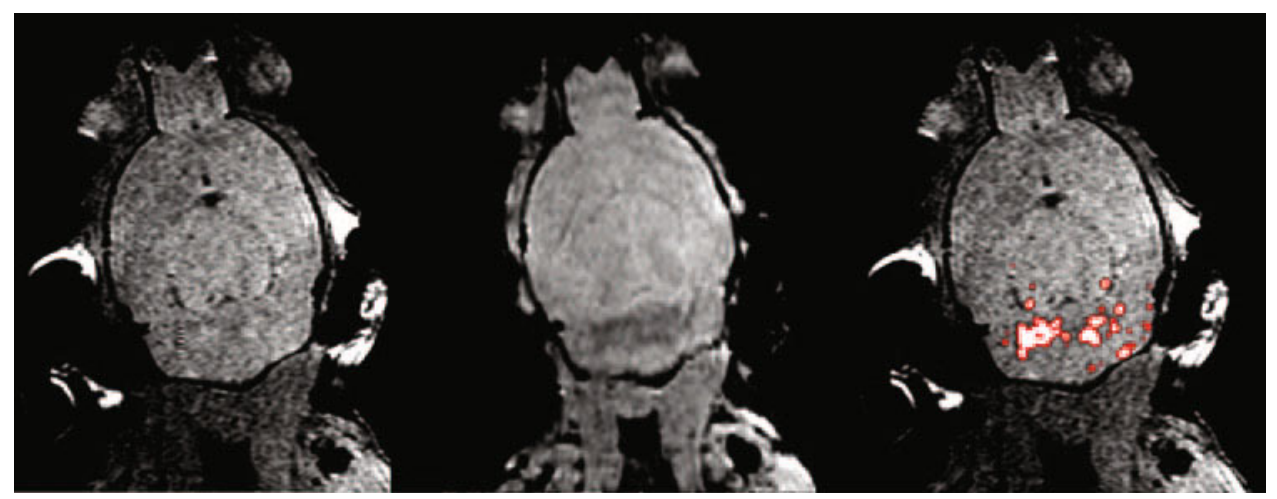

FIG. 7. $\mathrm{CD}^{+}$T-cell labeling with USPIO conjugated antibodies in Theiler's encephalitis virus infection. Left image: T1-weighted study shows faint enhancing areas in cerebellum. Middle image: T2*-weighted image shows hypointensities in cerebellum. Right image: composite image. A mask was generated by dividing the T1-weighted image with the T2* image, using an image algebra algorithm. Because the presence of USPIO is associated with slight enhancement on T1-weighted images and with marked hypointensity on T2*, by dividing the numeric intensity values depicting each voxel on the T1 and T2* images, the output will contain very high values in areas of high T1 and low T2* values. The generated mask was color-coded and superimposed on the T1-weighted image to clearly show the location and distribution of labeled cells. 

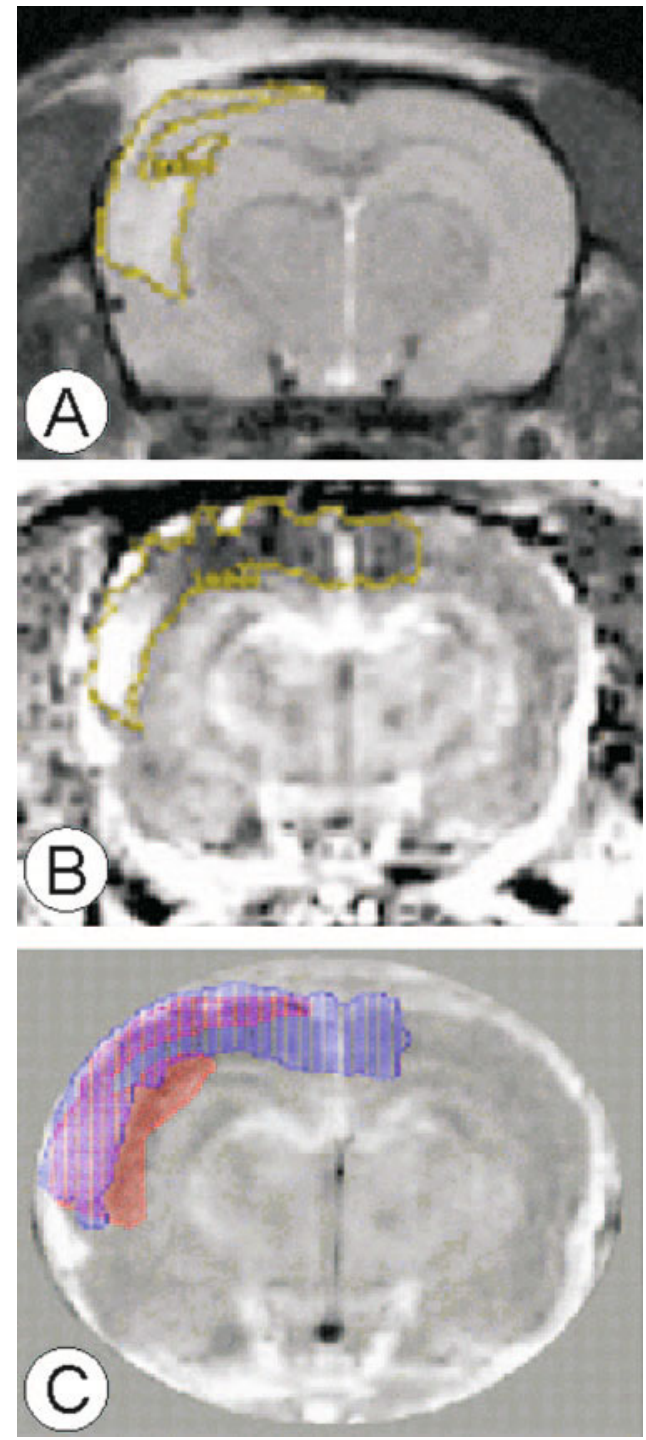

FIG. 8. Diffusion- and perfusion-weighted MRI studies in a rodent model. A: Diffusion-weighted image. B: Perfusion image. C: Diffusion-perfusion mismatch. The area of diffusion-perfusion mismatch is thought to represent potentially salvageable areas in stroke and brain injury models.

embryonic development have also been generated and reported. ${ }^{28}$ A web-based application assembled by the California Institute of Technology allows insight into one of these databases (http://mouseatlas.caltech.edu). Examples of in vivo and ex vivo anatomical imaging is shown on Figures 3 and 4.

In basic neuroscience research, tract tracing is an important development in experimental MRI methods. This technique utilizes paramagnetic $\mathrm{Mn}^{++}$ions. Neurons pass the $\mathrm{Mn}^{++}$ions to each other via the synaptic junctures, thus allowing visualization of neural pathways by MRI. ${ }^{29-32}$ It is believed that $\mathrm{Mn}^{++}$ions enter the cells due to receptor activated calcium channel opening. Once inside the cell, $\mathrm{Mn}^{++}$ions use axonal microtubule transport mechanisms to get to the axon terminal. During synaptic transmission, $\mathrm{Mn}^{++}$is released from the presynaptic neuron. It is able to enter the postsynaptic neuron via calcium channels. ${ }^{30}$ Thus, $\mathrm{Mn}^{++}$will accumulate in activated chains of neurons, leading to increased signal intensity in activated parts of the CNS. If applied topically to parts of the cortex, to olfactory neurons, or to the retina, $\mathrm{Mn}^{++}$can be used for tract tracing. While this technique requires invasive administration of this novel contrast material, the possibility of in vivo tract tracing is in itself a remarkable achievement.

Functional MRI studies can also be conducted in small rodents. fMRI studies are based on the blood oxygen level-dependent (BOLD) principle. ${ }^{2}$ Blood flow and oxy/ deoxy-hemoglobin related changes in activated areas of the cortex produce mild hypointensity on $\mathrm{T} 2 *$ images. ${ }^{33-36}$ Images obtained in the activated and non-activated state can be used to generate activity maps, including sensory or visual cortex mapping in animals. ${ }^{35,37}$ One potential limitation shown by several studies is that inhaled anesthetics may alter the signal. Also, mild hypocapnia may be helpful in establishing BOLD contrast. Some rodent fMRI studies also used SPIO/USPIO contrast materials to enhance signal loss at the activated areas ${ }^{36}$ these techniques also allow the measurement of cerebral blood volume. ${ }^{36,38}$

Immune-mediated diseases of the CNS are frequently studied in animal models. The most common models include experimental allergic encephalomyelitis (EAE), Theiler's Murine Encephalitis Virus infection (EV) and toxic demyelination models. For a review on imaging of (human) multiple sclerosis, the reader is referred to a separate article from the current volume. ${ }^{39}$ Similar to human diseases, lesion formation can be monitored by T2-weighted sequences (FIG. 5).

Gadolinium enhancement can be used to monitor the blood-brain barrier permeability associated with new lesion formation. ${ }^{40}$ Atrophy measurements become important for the chronic aspects of these diseases, volumetric MRI techniques based on 3D acquisition sequences ${ }^{40,41}$ are key components in the study of brain atrophy. Diffusion-weighted imaging (DWI) is also important in these models because this technique may show lesion formation even earlier than gadolinium contrast imaging. ${ }^{42}$ By the use of diffusion tensor imaging (DTI) methods, tract integrity can also be studied. ${ }^{43}$ Voxel based MRS (see below) or chemical shift imaging studies are also used, mainly to assess axonal integrity and membrane turnover. ${ }^{41,44}$

Several groups have reported success with immune cell-specific imaging, using superparamagnetic contrast materials (SPIO and USPIO) that are either internalized by cells after ex vivo incubation, or bound to the cells by specific antibodies ${ }^{45-50}$ (FIGS. 6 and 7). Another potential application of similar USPIO-based labeling techniques is to follow the distribution of biologically active 

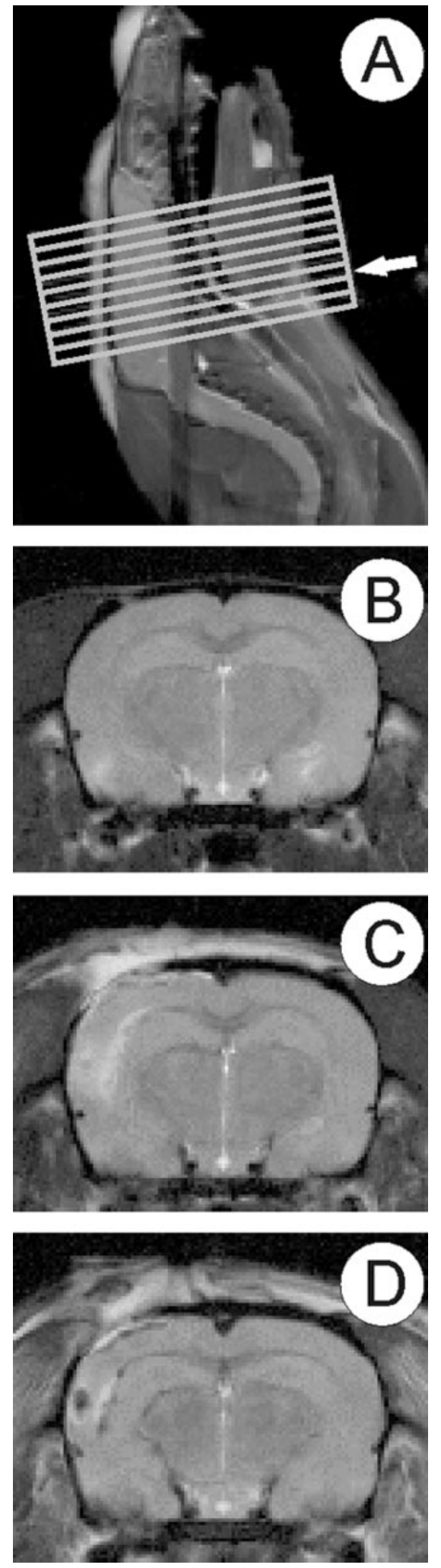

FIG. 9. Diffusion-weighted longitudinal imaging time course study of rat brain before and following traumatic brain injury. Sagittal slice (A) showing position of coronal slices. Coronal slice (B) before injury; coronal slice $24 \mathrm{~h}$ after injury (C) and $96 \mathrm{~h}$ after injury (D). Images C and D were obtained without the use of scout images and without the use of slice localization, by using a stereotactic probe designed at Georgetown University (FIG. 1.)

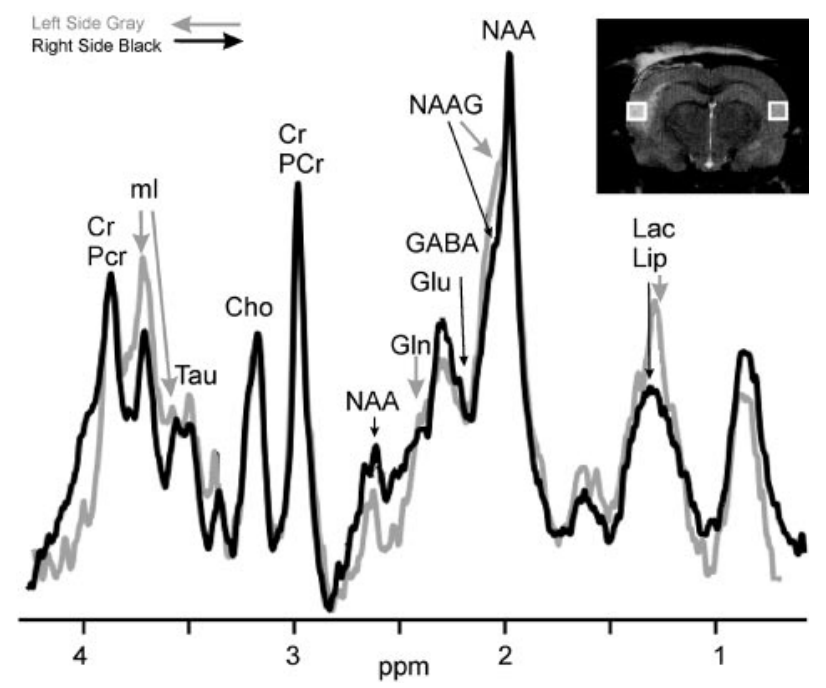

FIG. 10. In vivo spectra from left and right side of rat brain following traumatic brain injury. A single $8-\mu$ l voxel was positioned on lesion and another on the contralateral side. Differences in lactate, glutamate, NAAG, NAA, and myo-inositol are evident on in vivo MRS.

proteins. ${ }^{49}$ Our team successfully labeled remyelinating antibodies with this method. Such studies could lead to a much better understanding of immune-mediated diseases by allowing for real time tissue monitoring of inflammatory infiltration, replacing tedious and costly tissue manipulation techniques.

Cell-specific and molecular imaging studies are now used in the study of several disease models. ${ }^{49-51}$ In the near future, a surge of these techniques is expected, as the correlation between conventional histology and MRI methods is best achieved by the use of these tools.

In the study of neurodegenerative disease models, morphometric studies can be used for atrophy measurements. Both global brain atrophy measurements as well as focal volume loss of brain structures can be assessed using MRI. ${ }^{52-55}$ Iron deposition can also be studied. Because iron is known to cause susceptibility-related artifacts and high-field strength imaging is more prone to those artifacts (see above); thus, micro-MRI is a very convenient way to study this phenomenon. ${ }^{54}$ Alzheimer's pathology has been very difficult to study with MRI. However, through the use of novel contrast agents, one group has reported good success in imaging Alzheimer plaques ex vivo. ${ }^{56,57}$ More recently, even without contrast agents, plaque imaging in small rodent models has become possible both ex vivo ${ }^{58}$ and in vivo. ${ }^{59}$ MRS studies are also frequently used in the study of neurodegenerative diseases, mainly to assess axonal pathology by studying the NAA (N-acetyl aspartate) peak. ${ }^{60}$ Phosphorous MRS studies can also be used to study energy metabolites. $^{61}$

Experimental stroke models in small rodents are frequently studied with MRI methods. These generally re- 

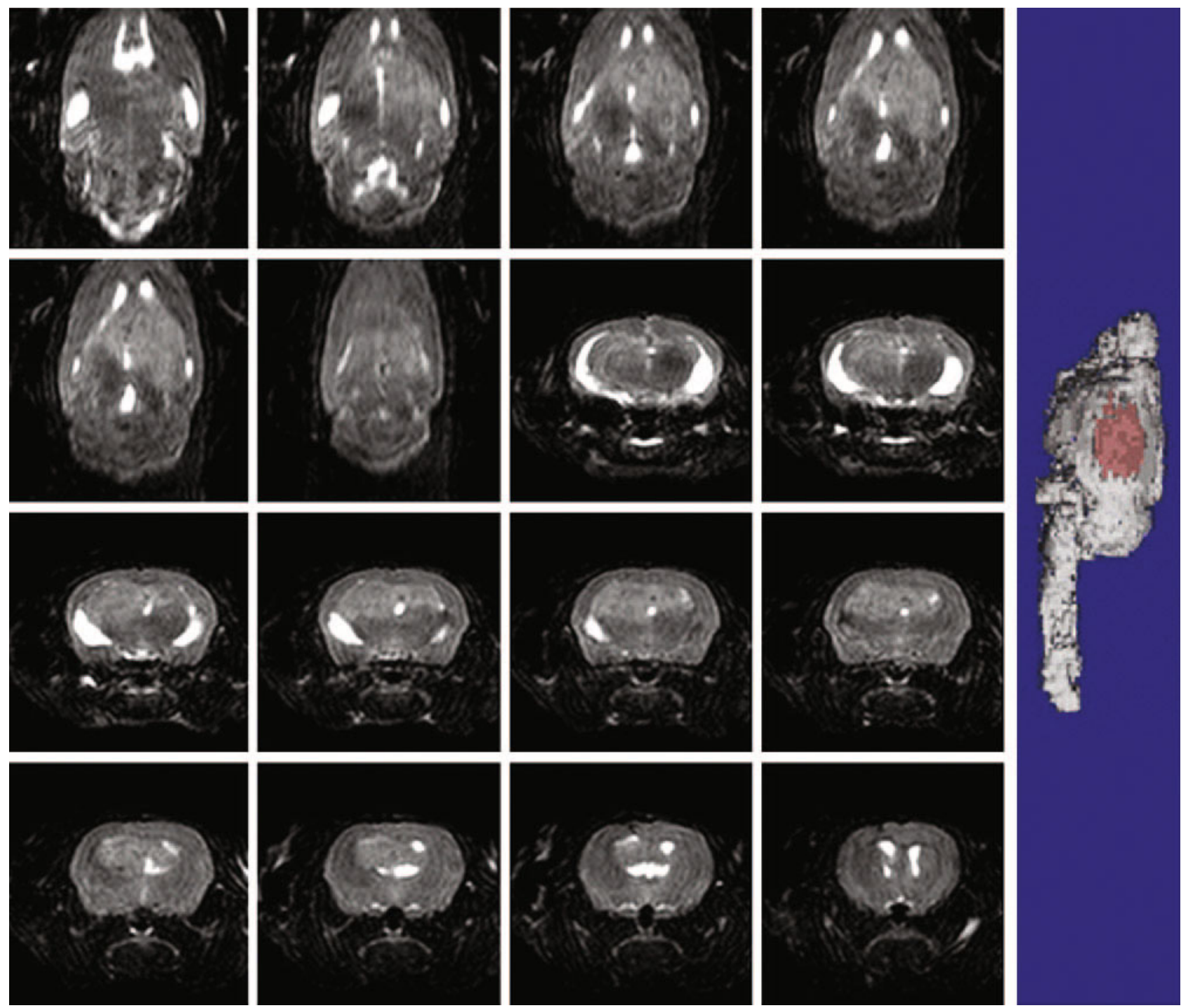

FIG. 11. Brain tumor imaging in nude mouse. ${ }^{83}$ Left panel: T2-weighted in vivo data set showing tumor growth on axial and coronal images. Note infiltrating tumor mass in thalamus and basal ganglia. Right panel: Semitransparent 3D rendering of the same data set. Using advanced post-processing methods, the 3D data set was segmented based on the signal characteristics from healthy brain tissue versus tumor-infiltrated tissue.

quire classic MR imaging modalities, including T1, T2, and proton density imaging. Newer techniques include the use of diffusion and perfusion imaging, also MRS for determining certain metabolites. ${ }^{62,63}$ Many groups have used experimental MCA ligation combined with hypoxia in rats or mice as models for human stroke. ${ }^{64}$ DWI sequences are especially powerful in detecting early ischemia, and many studies have used this resource in animal imaging, along with perfusion studies that are capable of visualizing the penumbra - the area at risk for infarction that potentially can be salvaged (FIG. 8). ${ }^{63,65}$

When DTI is done with a fast imaging sequence like EPI, the temporal resolution of scanning can be as low as every 20 - to 30 -s/entire brain scan, allowing close monitoring of stroke development. MRS studies have also been conducted, mainly looking at the lactate peak, which becomes prominent when anaerobic glycolysis predominates. ${ }^{62,63,66}$ Another important aspect is the NAA peak that may show decrease in completed strokes, but may remain normal if the animals still can recover from the ischemic event. ${ }^{67} \mathrm{MRA}^{68}$ and cerebral blood volume/cerebral blood flow studies ${ }^{38}$ have been done and evaluated in several stroke models. These studies can accurately characterize the blood flow and blood volume during ischemia.

Several models of brain trauma have been developed in experimental animals. These range from diffuse injuries to controlled cortical impacts, chemical insult, nonimpact models. MRI allows monitoring of the damaged tissue, also the tissue at risk. ${ }^{11,69,70}$ For this purpose, classic T2/T1/PD (proton density) methods have been used, along with the more accurate and more sensitive 


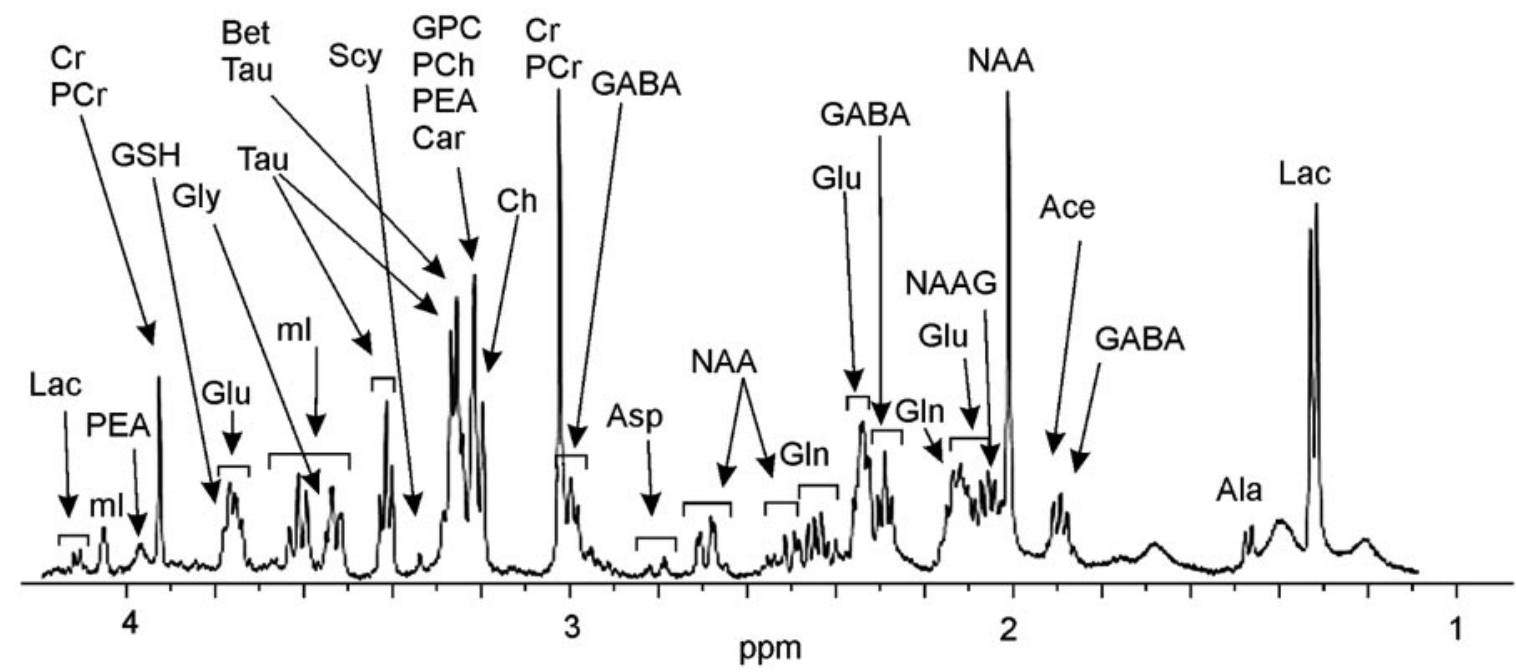

FIG. 12. High-resolution magic angle spinning (HR-MAS) NMR spectroscopy results of ex vivo brain sample at 11.7 Tesla.

DWI/DTI/MT (magnetization transfer) techniques, similarly to stroke studies described above (FIG. 9) MR spectroscopy is also used for the study of tissue injury in the CNS (FIG. 10). ${ }^{71,72}$

Animal models of brain tumors are also frequently studied by MRI and MRS (FIG. 11). ${ }^{73-75}$ The basic T1and T2-weighted techniques may not always allow an obvious delineation between healthy and tumor infiltrated tissue. ${ }^{76}$

The use of intravenous contrast materials may show blood brain barrier breakdown in certain tumor models; also MRS studies (proton and phosphorous) can be used to study biochemical differences between cancerous and normal tissue in vivo. Diffusion and perfusion studies, along with blood flow and blood oxygen level-dependent studies ${ }^{77}$ may also provide useful information because tumor cells generally show increased metabolism due to their rapidly dividing nature, and thus they generally require higher blood flow and extract more oxygen from the blood compared to healthy tissue.

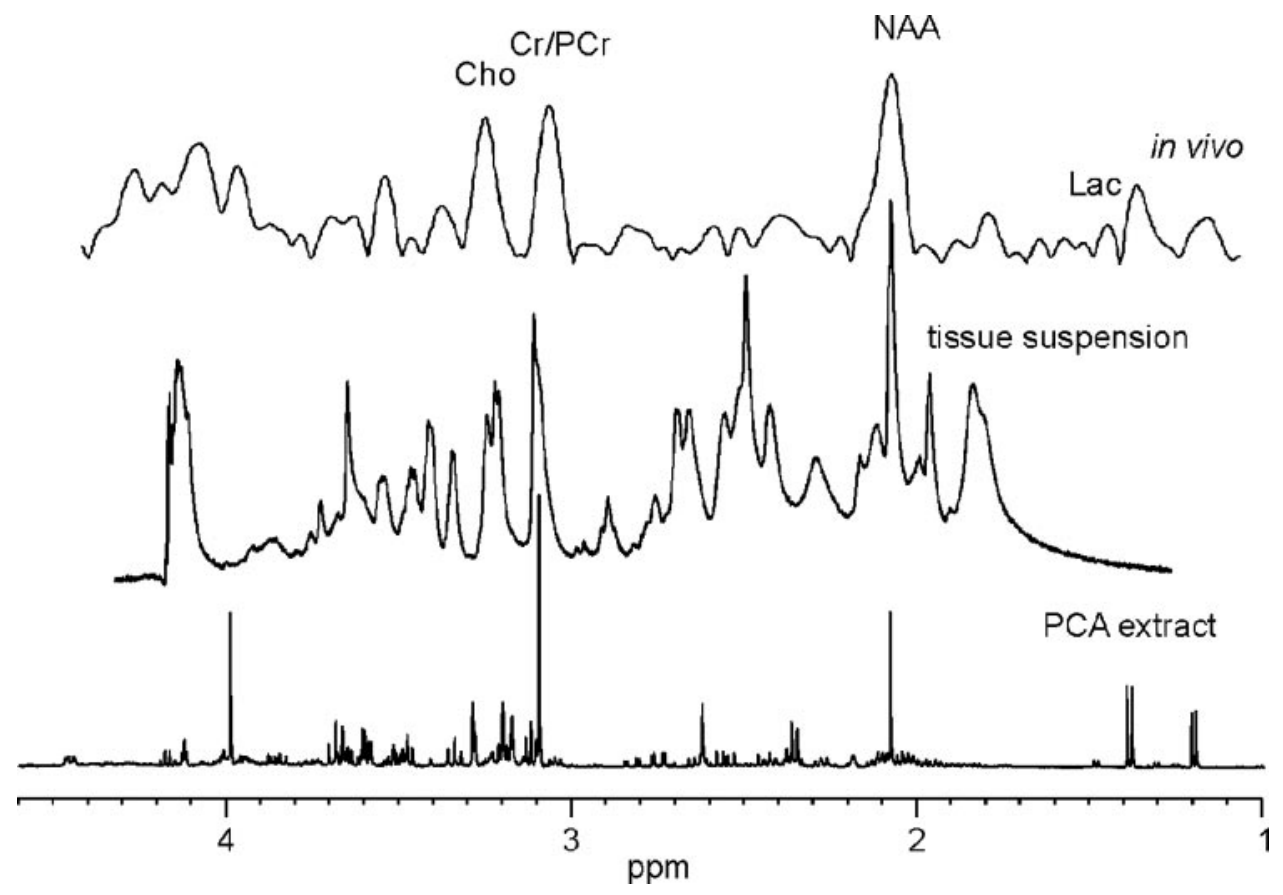

FIG. 13. Comparison of mouse brain $1 \mathrm{H}$ NMR spectra in vivo (7 T, $300 \mathrm{MHz})$, tissue suspension (11.4 T, $500 \mathrm{MHz})$ and PCA (perchloric acid) extract (11.4 T, $500 \mathrm{MHz}$ ). In vivo is biologically most relevant, but spectra suffer from poor resolution; PCA extracts provide extremely high resolution, for example, can resolve $\mathrm{Cr}$ and $\mathrm{CrP}$ lines, but the animal has to be sacrificed and extracts are cumbersome to prepare. Brain tissue suspension offers intermediate solution, resolution is modest but samples are easy to prepare. PCA extract spectra are somewhat different from the tissue suspension and in vivo spectra because PCA extracts only water-soluble metabolites. Thus, lipids and other water-insoluble components are absent in the PCA spectra. 


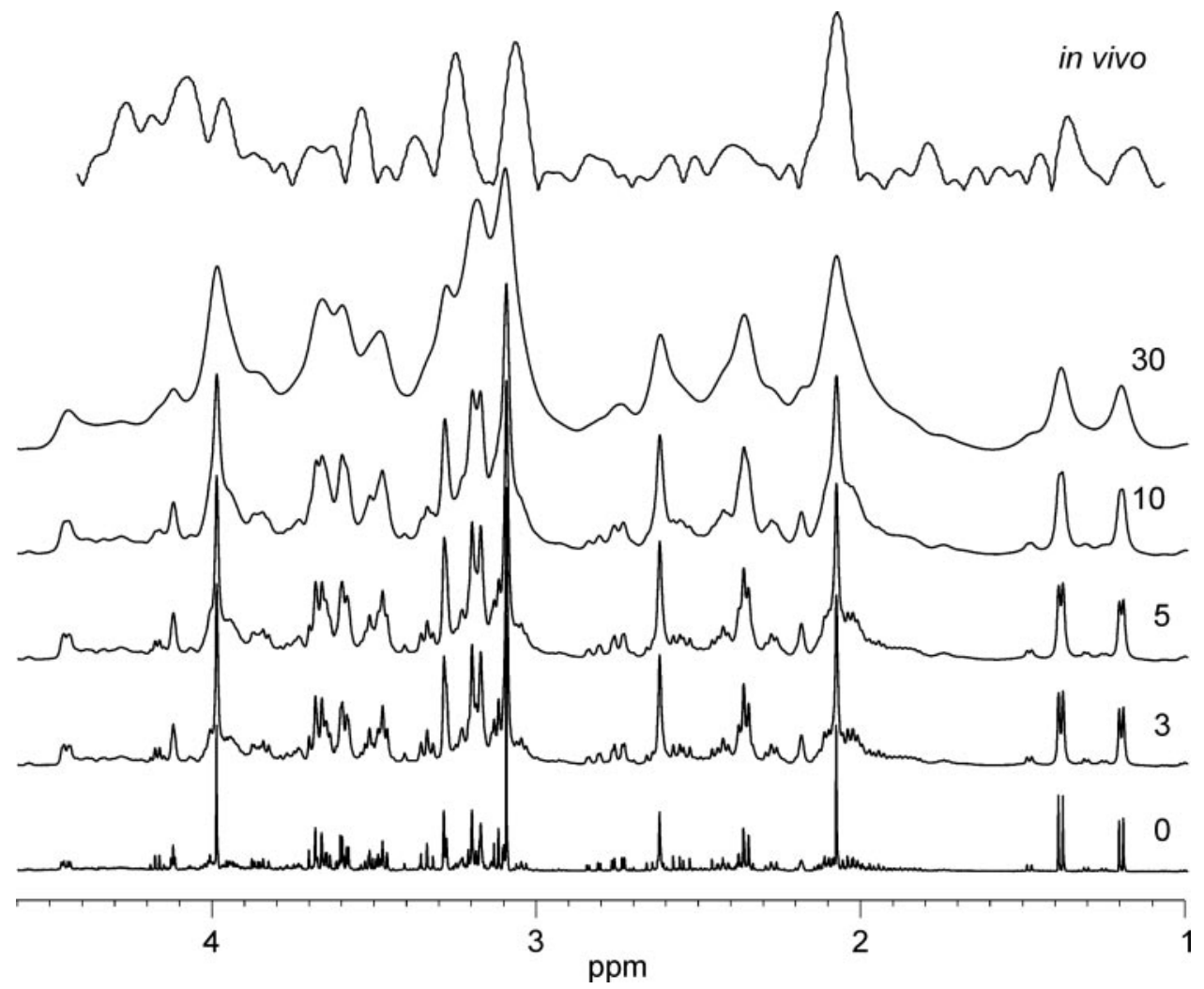

FIG. 14. In vivo mouse brain MRS and ex vivo PCA brain extract comparison, with various line broadenings (LB) applied to the PCA extract spectra as indicated on the right (in $\mathrm{Hz}$ ). Spectra are aligned at NAA line. Small chemical shift differences are caused by different sample temperatures $\left(22 \mathrm{C}\right.$ vs $\left.37^{\circ} \mathrm{C}\right)$ and $\mathrm{pH}$ (9 vs 7.2). In vivo spectrum roughly corresponds to $\mathrm{LB} 30 \mathrm{~Hz}$ ex vivo spectrum.

\section{Magnetic resonance spectroscopy}

In many of the above models of CNS diseases, MRS methods are frequently used to complement the information obtained by proton MRI imaging. ${ }^{1} \mathrm{H}$ MRS is a versatile noninvasive technique capable of producing information on a large number of brain chemicals in both humans $^{3}$ and animals. ${ }^{78}$ In addition to in vivo single voxel spectroscopy techniques, a newer development for

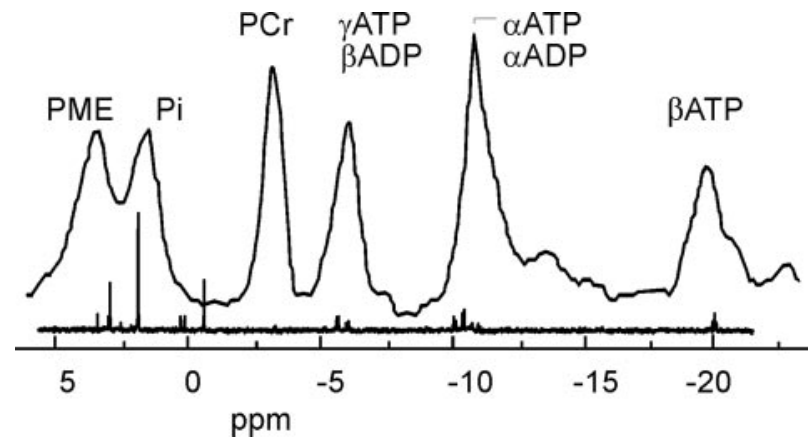

FIG. 15. ${ }^{31} \mathrm{P}$ spectrum of rat brain in vivo, in deep hypothermia. Spectrum was recorded using a 9.4 Tesla $(400 \mathrm{MHz}$ for protons or $161.9 \mathrm{MHz}$ for ${ }^{31} \mathrm{P}$ ) NMR spectrometer with surface coil and was baseline corrected to eliminate background signal from the skull. Acquisition time: $66 \mathrm{~s}$, NEX 64, TR $1 \mathrm{~s}$. Referenced with respect to $85 \% \mathrm{H}_{3} \mathrm{PO}_{4}, \mathrm{PCr}=-3.12 \mathrm{ppm}$. For reference, a $31 \mathrm{P}$ NMR spectrum of the normal mouse cord PCA extract recorded a 14.1 Tesla NMR spectrometer is also shown below the in vivo spectrum. in vivo MRS data acquisition is a technique called chemical shift imaging (CSI), ${ }^{79,80}$ which allows relatively rapid collection of spectra from several voxels describing an entire slice of the brain. For ex vivo samples from localized areas of the brain or from CSF, High Resolution Magic Angle Spinning (HR-MAS) techniques can be used to obtain very high resolution spectra (FIG. 12)..$^{81}$

To illustrate the difference in line resolution, different ex vivo and corresponding in vivo proton spectra are presented in Figure 13. In vivo proton spectra can be considered similar to brain suspension spectrum after line broadening, as shown in Figure 14.

It is important to understand that while none of the MRS visible metabolites are specific for one particular disease, MR spectra can provide a unique insight into the biochemical microenvironment of the studied voxel of interest. The following metabolites are studied most frequently with MRS in CNS diseases: NAA for its role in mitochondrial oxidative metabolism, as a putative marker for neuronal viability and its role in lipid synthesis (source of acetyl groups); $\mathrm{Cr}$ (Creatine) and $\mathrm{PCr}$ (phosphocreatine) as creatine to phosphocreatine energy conversion is mediated by creatine kinase; choline (Cho) as a precursor for neurotransmitter acetylcholine and membrane phospholipids, phosphatidylcholine and sphingomyelin; myo-inositol (mI) as a neuronal signal- 


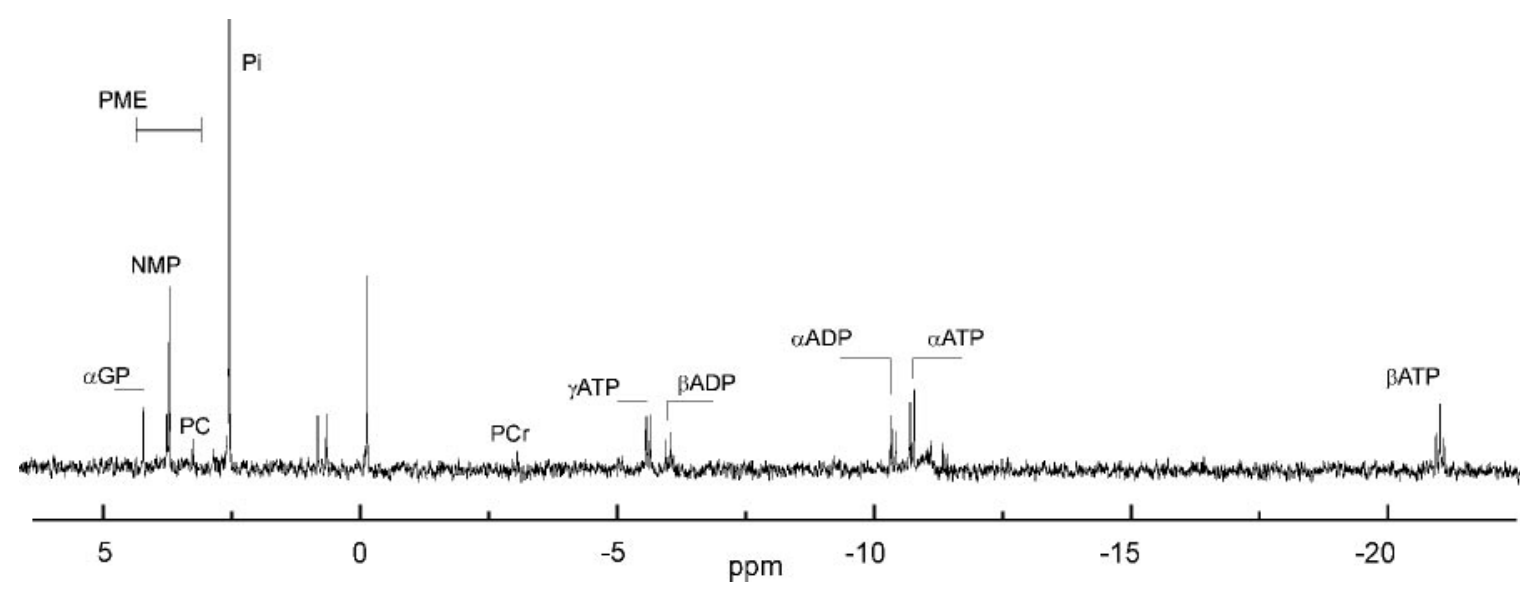

FIG. 16. ${ }^{31} \mathrm{P}$ spectra of mouse spinal cord PCA extracts recorded using a 11.4 Tesla NMR spectrometer. Within 1 min (at least 1 min is necessary to freeze the spinal cord after animal sacrifice) most of the PCr is gone; also notable are increase of ADP, $\alpha$-glycerophosphate ( $\alpha \mathrm{GP})$ and nucleotide monophosphates (NMP) (lines in phosphomonoester (PME) region). (G6P, glucoso-6-phosphate). Acquisition parameters: SF $242.9 \mathrm{MHz}$, NEX 8900, TR $5 \mathrm{~s}$, total time: $12 \mathrm{~h}$.

ing of the phosphoinositide pathway, osmoregulation, cell nutrition, and detoxification; lactate (Lac) which is a byproduct of anaerobic metabolism, its elevated concentrations result form glycolytic metabolism as happens in brain ischemia; glutamate (Glu) and glutamine (Gln), which are important neurotransmitters in the CNS.

${ }^{31} \mathrm{P}$ (Phosphorous) MRS can also be performed in vivo (FIG. 15). ${ }^{61}$ While the spatial resolution with this technique is lower than that of proton MRS, phosphorous MRS can provide important information about energy metabolites, including inorganic phosphorous, phosphocreatine, and $\operatorname{ATP}(\gamma, \alpha$, and $\beta$ peaks) (FIG. 16); it can also be used for in vivo $\mathrm{pH}$ measurement. ${ }^{82}$

\section{CONCLUSION}

Over the last several years, microscopic resolution MRI has become a popular and versatile tool in the study of CNS diseases. The driving force behind this enormous growth is related to the development of improved MRI hardware and software and to the development of different transgenic and knockout rodent models of CNS diseases. MR image resolution of tens of micrometers in three dimensions can now be achieved for in vivo studies. Because of the obstacles encountered with physiological microscopic movements of tissues due to circulation, breathing, fluid flow, significantly higher resolutions are probably unrealistic for in vivo applications. Newer application development is aimed toward higher temporal resolution. This will be used to study different time points of the disease process and for oversampling, which will lead to improved SNR. New methods are continuously under development to provide improved SNR and especially CNR, allowing for better differentiation between tissues and cells in vivo. Cell-specific and molecular imaging techniques can be considered as specific techniques designed to improve the CNR toward a certain tissue, cell type, or receptor of interest. An abundance of new applications that use and advance these targeted contrast techniques is expected. The number of universities and research institutions owning small animal systems will likely continue to grow. The shift in science toward in vivo dynamic imaging may also result in a reduction in the use of conventional histology-based techniques, which in turn may reduce the number of animals necessary for experiments. The increased versatility that microscopic resolution MRI is beginning to provide will lead to new insight into the processes that determine life and death and should result in the development of new diagnostic and treatment approaches in several human diseases.

Acknowledgments: The authors would like to acknowledge ASI Instruments Inc. in Warren, MI, for the building and donating to the construction of the presented mouse holder (FIG. 1). The animal models of traumatic brain injury are from the lab of Dr. Ibolja Cernak, Associate Professor, Department of Neuroscience, Georgetown University, Washington, DC. The diffusion-perfusion renderings were preformed by Dr. John W. VanMeter, Assistant Professor, Department of Neurology, Georgetown University, Washington, DC. The presented brain tumor model is from the laboratory of Dr. C. David James, Dr. Evanthia X. Galanis, Dr. Jann N. Sarkaria, and Dr. Brian O'Neill, Mayo Clinic College of Medicine (Rochester, MN).

\section{REFERENCES}

1. Hansen G, Crooks LE, Davis P, De Groot J, Herfkens R, Margulis $\mathrm{AR}$, et al. In vivo imaging of the rat anatomy with nuclear magnetic resonance. Radiology 136:695-700, 1980.

2. Bammer R, Skare S, Newbould R, Liu C, Thijs V, Ropele S, Clayton DB, Krueger G, Moseley ME, Glover GH. Foundations of advanced magnetic resonance imaging. NeuroRx 2:167-196, 2005.

3. Lin A, Ross BD, Harris K, Wong W. Efficacy of proton magnetic resonance spectroscopy in neurological diagnosis and neurotherapeutic decision making. NeuroRx 2:197-214, 2005.

4. Brooks DJ. Positron emission tomography and single-photon emission computed tomography in central nervous system drug development. NeuroRx 2:226-236, 2005. 
5. Devous MD Sr. Single-photon emission computed tomography in neurotherapeutics. NeuroRx 2:237-249, 2005.

6. Robb RA. The virtualization of medicine: a decade of pitfalls and progress. Stud Health Technol Inform 85:1-7, 2002.

7. Robb RA. 3-D visualization in biomedical applications. Аnnu Rev Biomed Eng 1:377-399, 1999.

8. Turner R, Howseman A, Rees GE, Josephs O, Friston K. Functional magnetic resonance imaging of the human brain: data acquisition and analysis. Exp Brain Res 123:5-12, 1998.

9. Lukasik VM, Gillies RJ. Animal anaesthesia for in vivo magnetic resonance. NMR Biomed 16:459-467, 2003.

10. Kannurpatti SS, Biswal BB. Effect of anesthesia on CBF, MAP and fMRI-BOLD signal in response to apnea. Brain Res 1011:141147, 2004.

11. Fricke ST, Vink R, Chiodo C, Cernak I, Ileva L, Faden AI. Consistent and reproducible slice selection in rodent brain using a novel stereotaxic device for MRI. J Neurosci Methods 136:99102, 2004.

12. Haacke EM, Brown RW, Thompson MR, Venkatesan R. Magnetic resonance imaging: physical principles and sequence design. Ed 1. Canada: Wiley-Liss, 1999.

13. McRobbie DW, Moore EA, Graves MJ, Prince MR. MRI: from picture to protons. Cambridge, UK: Cambridge University Press, 2002.

14. Liang Z-P, Lauterbur PC. Principles of magnetic resonance imaging: a signal processing perspective. Ed 1. Piscataway, NJ: WileyIEEE Press, 1999.

15. Kumar A, Welti D, Ernst RR. NMR Fourier zeugmatography. $J$ Magn Reson 18:69-83, 1975.

16. Ernst RR. Nobel Lecture. Nuclear magnetic resonance Fourier transform spectroscopy. Biosci Rep 12:143-187, 1992.

17. Back PJ, Coy A, Xia Y, Callaghan PT, Diamante LM, Umbach SL. Some biophysical applications of motional contrast in n.m.r. microscopy. Int J Biol Macromol 13:181-189, 1991.

18. Callaghan PT, Clark CJ, Forde LC. Use of static and dynamic NMR microscopy to investigate the origins of contrast in images of biological tissues. Biophys Chem 50:225-235, 1994.

19. Yang QX, Williams GD, Demeure RJ, Mosher TJ, Smith MB. Removal of local field gradient artifacts in $\mathrm{T} 2 *$-weighted images at high fields by gradient-echo slice excitation profile imaging. Magn Reson Med 39:402-409, 1998.

20. Yang QX, Demeure RJ, Dardzinski BJ, Arnold BW, Smith MB. Multiple echo frequency-domain image contrast: improved signalto-noise ratio and T2 (T2*) weighting. Magn Reson Med 41:423428, 1999.

21. Ordidge RJ, Gorell JM, Deniau JC, Knight RA, Helpern JA. Assessment of relative brain iron concentrations using T2-weighted and T2*-weighted MRI at 3 Tesla. Magn Reson Med 32:335-341, 1994.

22. Frahm J, Merboldt KD, Hanicke W. Direct FLASH MR imaging of magnetic field inhomogeneities by gradient compensation. Magn Reson Med 6:474-480, 1988.

23. Hennig J, Friedburg H. Clinical applications and methodological developments of the RARE technique. Magn Reson Imaging 6:391-395, 1988.

24. Jacobs RE, Papan C, Ruffins S, Tyszka JM, Fraser SE. MRI: volumetric imaging for vital imaging and atlas construction. Nat Rev Mol Cell Biol Suppl:S10-S16, 2003.

25. Dhenain M, Ruffins SW, Jacobs RE. Three-dimensional digital mouse atlas using high-resolution MRI. Dev Biol 232:458-470, 2001.

26. MacKenzie-Graham A, Lee EF, Dinov ID, Bota M, Shattuck DW, Ruffins $\mathrm{S}$ et al. A multimodal, multidimensional atlas of the C57BL/6J mouse brain. J Anat 204:93-102, 2004.

27. Kovacevic N, Henderson JT, Chan E, Lifshitz N, Bishop J, Evans $\mathrm{AC}$, et al. A three-dimensional MRI atlas of the mouse brain with estimates of the average and variability. Cereb Cortex, September 1, 2004.

28. Jacobs RE, Ahrens ET, Dickinson ME, Laidlaw D. Towards a microMRI atlas of mouse development. Comput Med Imaging Graph 23:15-24, 1999.

29. Pautler RG, Mongeau R, Jacobs RE. In vivo trans-synaptic tract tracing from the murine striatum and amygdala utilizing manganese enhanced MRI (MEMRI). Magn Reson Med 50:33-39, 2003.

30. Pautler RG, Koretsky AP. Tracing odor-induced activation in the olfactory bulbs of mice using manganese-enhanced magnetic resonance imaging. NeuroImage 16:441-448, 2002.

31. Hu TC, Pautler RG, MacGowan GA, Koretsky AP. Manganeseenhanced MRI of mouse heart during changes in inotropy. Magn Reson Med 46:884-890, 2001.

32. Pautler RG, Silva AC, Koretsky AP. In vivo neuronal tract tracing using manganese-enhanced magnetic resonance imaging. Magn Reson Med 40:740-748, 1998.

33. Malisza KL, Stroman PW. Functional imaging of the rat cervical spinal cord. J Magn Reson Imaging 16:553-558, 2002.

34. Malisza KL, Stroman PW, Turner A, Gregorash L, Foniok T, Wright A. Functional MRI of the rat lumbar spinal cord involving painful stimulation and the effect of peripheral joint mobilization. J Magn Reson Imaging 18:152-159, 2003.

35. Bock C, Schmitz B, Kerskens CM, Gyngell ML, Hossmann KA, Hoehn-Berlage M. Functional MRI of somatosensory activation in rat: effect of hypercapnic up-regulation on perfusion- and BOLDimaging. Magn Reson Med 39:457-461, 1998.

36. Mandeville JB, Marota JJ, Kosofsky BE, Keltner JR, Weissleder $\mathrm{R}$, Rosen BR et al. Dynamic functional imaging of relative cerebral blood volume during rat forepaw stimulation. Magn Reson Med 39:615-624, 1998.

37. Brinker G, Bock C, Busch E, Krep H, Hossmann KA, HoehnBerlage M. Simultaneous recording of evoked potentials and T2*weighted MR images during somatosensory stimulation of rat. Magn Reson Med 41:469-473, 1999.

38. Wu EX, Wong KK, Andrassy M, Tang H. High-resolution in vivo CBV mapping with MRI in wild-type mice. Magn Reson Med 49:765-770, 2003.

39. Bakshi R, Minagar A, Jaisani Z, Wolinsky JS. Imaging of multiple sclerosis: role in neurotherapeutics. NeuroRx 2:277-303.

40. Pirko I, Gamez J, Johnson AJ, Macura SI, Rodriguez M. Dynamics of MRI lesion development in an animal model of viral-induced acute progressive CNS demyelination. Neuroimage 21:576-582, 2004.

41. Pirko I, Johnson A, Gamez J, Macura SI, Rodriguez M. Disappearing "T1 black holes" in an animal model of multiple sclerosis. Front Biosci 9:1222-1227, 2004.

42. Heide AC, Richards TL, Alvord EC Jr, Peterson J, Rose LM Diffusion imaging of experimental allergic encephalomyelitis. Magn Reson Med 29:478-484, 1993.

43. Ahrens ET, Laidlaw DH, Readhead C, Brosnan CF, Fraser SE, Jacobs RE. MR microscopy of transgenic mice that spontaneously acquire experimental allergic encephalomyelitis. Magn Reson Med 40:119-132, 1998.

44. Schwarcz A, Natt O, Watanabe T, Boretius S, Frahm J, Michaelis T. Localized proton MRS of cerebral metabolite profiles in different mouse strains. Magn Reson Med 49:822-827, 2003.

45. Bulte JW, Kraitchman DL. Iron oxide MR contrast agents for molecular and cellular imaging. NMR Biomed 17:484-499, 2004.

46. Bulte JW, Arbab AS, Douglas T, Frank JA. Preparation of magnetically labeled cells for cell tracking by magnetic resonance imaging. Methods Enzymol 386:275-299, 2004.

47. Bulte JW, Ben-Hur T, Miller BR, Mizrachi-Kol R, Einstein O, Reinhartz E, et al. MR microscopy of magnetically labeled neurospheres transplanted into the Lewis EAE rat brain. Magn Reson Med 50:201-205, 2003.

48. Anderson SA, Shukaliak-Quandt J, Jordan EK, Arbab AS, Martin $\mathrm{R}$, McFarland $\mathrm{H}$, et al. Magnetic resonance imaging of labeled T-cells in a mouse model of multiple sclerosis. Ann Neurol 55: 654-659, 2004.

49. Pirko I, Ciric B, Gamez J, Bieber AJ, Warrington AE, Johnson AJ, et al. A human antibody that promotes remyelination enters the CNS and decreases lesion load as detected by T2-weighted spinal cord MRI in a virus-induced murine model of MS. FASEB $J$ 18:1577-1579, 2004.

50. Pirko I, Ciric B, Johnson AJ, Gamez J, Rodriguez M, Macura S. Magnetic resonance imaging of immune cells in inflammation of central nervous system. Croat Med J 44:463-468, 2003. 
51. Pirko I, Johnson A, Ciric B, Gamez J, Macura SI, Pease LR, et al. In vivo magnetic resonance imaging of immune cells in the central nervous system with superparamagnetic antibodies. FASEB $J$ 18: 179-182, 2004.

52. Lee WT, Chang C. Magnetic resonance imaging and spectroscopy in assessing 3-nitropropionic acid-induced brain lesions: an animal model of Huntington's disease. Prog Neurobiol 72:87-110, 2004.

53. Choi IY, Lee SP, Guilfoyle DN, Helpern JA. In vivo NMR studies of neurodegenerative diseases in transgenic and rodent models. Neurochem Res 28:987-1001, 2003.

54. Gilissen EP, Ghosh P, Jacobs RE, Allman JM. Topographical localization of iron in brains of the aged fat-tailed dwarf lemur (Cheirogaleus medius) and gray lesser mouse lemur (Microcebus murinus). Am J Primatol 45:291-299, 1998.

55. McDaniel B, Sheng H, Warner DS, Hedlund LW, Benveniste H. Tracking brain volume changes in C57BL/6J and ApoE-deficient mice in a model of neurodegeneration: a 5-week longitudinal micro-MRI study. Neuroimage 14:1244-1255, 2001.

56. Poduslo JF, Wengenack TM, Curran GL, Wisniewski T, Sigurdsson EM, Macura SI, et al. Molecular targeting of Alzheimer's amyloid plaques for contrast-enhanced magnetic resonance imaging. Neurobiol Dis 11:315-329, 2002.

57. Poduslo JF, Curran GL, Peterson JA, McCormick DJ, Fauq AH, Khan MA, et al. Design and chemical synthesis of a magnetic resonance contrast agent with enhanced in vitro binding, high blood-brain barrier permeability, and in vivo targeting to Alzheimer's disease amyloid plaques. Biochemistry 43:6064-6075, 2004.

58. Zhang J, Yarowsky P, Gordon MN, Di Carlo G, Munireddy S, van Zijl PC, et al. Detection of amyloid plaques in mouse models of Alzheimer's disease by magnetic resonance imaging. Magn Reson Med 51:452-457, 2004.

59. Jack CR Jr, Garwood M, Wengenack TM, Borowski B, Curran GL, Lin J, et al. In vivo visualization of Alzheimer's amyloid plaques by magnetic resonance imaging in transgenic mice without a contrast agent. Magn Reson Med 52:1263-1271, 2004.

60. Dedeoglu A, Choi JK, Cormier K, Kowall NW, Jenkins BG. Magnetic resonance spectroscopic analysis of Alzheimer's disease mouse brain that express mutant human APP shows altered neurochemical profile. Brain Res 1012:60-65, 2004.

61. Andjus RK, D'akula A, Markley JL, Macura S. Brain energetics and tolerance to anoxia in deep hypothermia. Ann NY Acad Sci, in press.

62. Hesselbarth D, Franke C, Hata R, Brinker G, Hoehn-Berlage M. High resolution MRI and MRS: a feasibility study for the investigation of focal cerebral ischemia in mice. NMR Biomed 11:423$429,1998$.

63. Hoehn M, Nicolay K, Franke C, van der Sanden B. Application of magnetic resonance to animal models of cerebral ischemia. J Magn Reson Imaging 14:491-509, 2001.

64. Hata R, Mies G, Wiessner C, Fritze K, Hesselbarth D, Brinker G, et al. A reproducible model of middle cerebral artery occlusion in mice: hemodynamic, biochemical, and magnetic resonance imaging. J Cereb Blood Flow Metab 18:367-375, 1998.

65. Xue R, van Zijl PC, Crain BJ, Solaiyappan M, Mori S. In vivo three-dimensional reconstruction of rat brain axonal projections by diffusion tensor imaging. Magn Reson Med 42:1123-1127, 1999.

66. Dreher W, Kuhn B, Gyngell ML, Busch E, Niendorf T, Hossmann $\mathrm{KA}$, et al. Temporal and regional changes during focal ischemia in rat brain studied by proton spectroscopic imaging and quantitative diffusion NMR imaging. Magn Reson Med 39:878-888, 1998.

67. Malisza KL, Kozlowski P, Ning G, Bascaramurty S, Tuor UI. Metabolite changes in neonatal rat brain during and after cerebral hypoxia-ischemia: a magnetic resonance spectroscopic imaging study. NMR Biomed 12:31-38, 1999.
68. Beckmann N. High resolution magnetic resonance angiography non-invasively reveals mouse strain differences in the cerebrovascular anatomy in vivo. Magn Reson Med 44:252-258, 2000.

69. Goetz P, Blamire A, Rajagopalan B, Cadoux-Hudson T, Young D, Styles P. Increase in apparent diffusion coefficient in normal appearing white matter following human traumatic brain injury correlates with injury severity. J Neurotrauma 21:645-654, 2004.

70. Stoffel M, Blau C, Reinl H, Breidt J, Gersonde K, Baethmann A, et al. Identification of brain tissue necrosis by MRI: validation by histomorphometry. J Neurotrauma 21:733-740, 2004.

71. Schuhmann MU, Stiller D, Skardelly M, Bernarding J, Klinge PM, Samii A et al. Metabolic changes in the vicinity of brain contusions: a proton magnetic resonance spectroscopy and histology study. J Neurotrauma 20:725-743, 2003.

72. Schuhmann MU, Stiller D, Skardelly M, Thomas S, Samii M, Brinker T. Long-time in-vivo metabolic monitoring following experimental brain contusion using proton magnetic resonance spectroscopy. Acta Neurochir Suppl 81:209-212, 2002.

73. Phuong LK, Allen C, Peng KW, Giannini C, Greiner S, TenEyck $\mathrm{CJ}$, et al. Use of a vaccine strain of measles virus genetically engineered to produce carcinoembryonic antigen as a novel therapeutic agent against glioblastoma multiforme. Cancer Res 63: 2462-2469, 2003.

74. Hirakawa K, Naruse S, Higuchi T, Horikawa Y, Tanaka C, Ebisu $\mathrm{T}$. The investigation of experimental brain tumours using 31PMRS and 1H-MRI. Acta Neurochir Suppl (Wien) 43:140-144, 1988.

75. Gyngell ML, Els T, Hoehn-Berlage M, Hossmann KA. Proton MR spectroscopy of experimental brain tumors in vivo. Acta Neurochir Suppl (Wien) 60:350-352, 1994.

76. Howe FA, Robinson SP, Rodrigues LM, Stubbs M, Griffiths JR. Issues in GRE \& SE magnetic resonance imaging to probe tumor oxygenation. Adv Exp Med Biol 530:441-448, 2003.

77. Howe FA, Robinson SP, McIntyre DJ, Stubbs M, Griffiths JR. Issues in flow and oxygenation dependent contrast (FLOOD) imaging of tumours. NMR Biomed 14:497-506, 2001.

78. Fricke S, Galloway M, Seraji-Bozorgzad N, Mitchell T, Posse S, More G. Ex-vivo neurochemical kinetics in brain tissue specimens monitored via quantative in-vivo MRS of the mouse brain. Paper presented at the conference of the International Society for Magnetic Resonance in Medicine, Honolulu, HI, p 159, 2002.

79. Pfeuffer J, Tkac I, Provencher SW, Gruetter R. Toward an in vivo neurochemical profile: quantification of 18 metabolites in shortecho-time (1)H NMR spectra of the rat brain. J Magn Reson 141:104-120, 1999.

80. Lenkinski RE, Holland GA, Allman T, Vogele K, Kressel HY, Grossman RI, et al. Integrated MR imaging and spectroscopy with chemical shift imaging of P-31 at $1.5 \mathrm{~T}$ : initial clinical experience. Radiology 169:201-206, 1988.

81. Pykett IL, Rosen BR. Nuclear magnetic resonance: in vivo proton chemical shift imaging. Work in progress. Radiology 149:197201, 1983.

82. Barton SJ, Howe FA, Tomlins AM, Cudlip SA, Nicholson JK, Bell BA et al. Comparison of in vivo $1 \mathrm{H}$ MRS of human brain tumours with 1H HR-MAS spectroscopy of intact biopsy samples in vitro. Magma 8:121-128, 1999.

83. Kamezawa T, Asakura T, Yatsushiro K, Niiro M, Kasamo S, Fujimoto T. 31P-magnetic resonance spectroscopic study on the effect of glycerol on cold-induced brain edema. Acta Neurochir Suppl (Wien) 60:499-501, 1994.

84. Giannini C, Sarkaria J, Saito A, Uhm J, Galanis E, Carlson B, et al. Patient tumor EGFR and PDGFRA gene amplifications retained in an invasive intracranial xenograft model of GBM. Neuro-Oncol$o g y$, in press. 